\title{
La « Théorie-pratique » du pisé.
}

Mise en discours et en images d'une technique et sa réception dans le Sud-Ouest de la France aux XVIII ${ }^{\mathrm{e}}$ et XIX ${ }^{\mathrm{e}}$ siècles

The "practice-theory" of pisé. Settings in words and pictures of a technique and how it was received in the southwest of France $\left(18^{\text {th }}-19^{\text {th }}\right.$ centuries)

$L a$ «Teorfa-práctica » del adobe (pisé). Puesta en discurso y en imágenes de una técnica y su recepción en el sureste de Francia durante los siglos XVIII-XIX

\section{Valérie Nègre}

\section{OpenEdition}

Journals

Édition électronique

URL : https://journals.openedition.org/tc/57

DOI : $10.4000 /$ tc. 57

ISSN : 1952-420X

Éditeur

Éditions de l'EHESS

Édition imprimée

Date de publication : 1 décembre 2003

Pagination : 47-63

ISSN : 0248-6016

Référence électronique

Valérie Nègre, «La « Théorie-pratique » du pisé. », Techniques \& Culture [En ligne], 41 | 2003, mis en ligne le 13 janvier 2005, consulté le 29 septembre 2022. URL : http://journals.openedition.org/tc/57 ; DOI : https://doi.org/10.4000/tc.57

Ce document a été généré automatiquement le 29 septembre 2022

Tous droits réservés 


\section{La « Théorie-pratique » du pisé.}

Mise en discours et en images d'une technique et sa réception dans le Sud-Ouest de la France aux XVIII ${ }^{\mathrm{e}}$ et $\mathrm{XIX}^{\mathrm{e}}$ siècles

The "practice-theory" of pisé. Settings in words and pictures of a technique and how it was received in the southwest of France $\left(18^{\text {th }}-19^{\text {th }}\right.$ centuries)

La « Teorfa-práctica » del adobe (pisé). Puesta en discurso y en imágenes de una técnica y su recepción en el sureste de Francia durante los siglos XVIII-XIX

Valérie Nègre

1 C'est à des agronomes et des architectes originaires de la région lyonnaise que l'on doit la « mise en règle » du pisé. Leurs enquêtes sont moins motivées par l'idée de contrôler les pratiques -éviter les «tromperies » des ouvriers- que par la volonté de diffuser et de perfectionner une technique à laquelle ils trouvent de nombreux avantages: économie, durabilité, isolation thermique, salubrité. Les agronomes insistent aussi sur l'excellent engrais que le pisé démoli constitue pour certaines cultures : une «merveille dans les vignes \& dans les terres à froment » (Goiffon $1772: 12$ ). Les architectes ajoutent à ces avantages I'incombustibilité du matériau, « l'art du pisé » est aussi pour eux « l'art de l'incombustibilité ».

\section{De la « boue » au pisé}

2 Le premier mémoire imprimé, entièrement consacré au pisé, est publié en 1772 par l'abbé Rozier dans son journal Observations sur la Physique, sur l'Histoire Naturelle \& sur les Arts et de manière indépendante par son auteur, Georges-Claude Goiffon, la même année, sous la forme d'un petit ouvrage intitulé L'Art du maçon piseur ${ }^{2}$. Pour la première fois sont choisis et explicités les termes propres à la technique, notamment le verbe, les substantifs et les adjectifs "piser, pisé, pison, piseur, pisé, pisée ", ainsi que les mots désignant les outils, tous empruntés, selon Goiffon, aux «maçons piseurs » ${ }^{3}$. Le texte décrit précisément les outils, les gestes et les opérations concernées et s'appuie sur une planche composée dans la tradition de la Description des Arts et Métiers: une vue 
perspective du bâtiment en cours de construction est associée à une panoplie d'outils représentés ici en géométral (vue latérale).

3 Mais l'essai de Goiffon n'est pas accueilli très favorablement par les Lyonnais. Le trouvant « ni assez clair ni assez méthodique », l'abbé Rozier s'adresse quelques années plus tard à Catherine-François Boulard ${ }^{4}$, pour rédiger l'article «Pisai ou pisé » de son Cours complet d'agricultur ${ }^{5}(1786, \mathrm{t}$. VII). Assujettit à des règles sûres des opérations qui dépendent de la justesse du coup d'oeil, du tour de main, n'est pas facile; pour l'architecte François Cointeraux, Goiffon manque avant tout de pratique personnelle ${ }^{6}$. Néanmoins, ces critiques ne remettent pas fondamentalement en cause son enquête. L'essai de Goiffon est repris et perfectionné.

4 L'architecte Boulard reprend, dans son très long article "Pisai ou pisé " sur le vocabulaire retenu par Goiffon et l'ensemble des dessins contenus dans sa planche. Le discours est cependant entièrement reconstruit, rédigé de manière plus claire et plus méthodique, selon l'ordre d'exécution des opérations; chaque étape de la réalisation étant mise en évidence par un sous-titre ${ }^{7}$. La planche est également complétée par deux représentations en plan du mur en cours de montage (fig. 1).

5 En 1790 et 1791 paraissent les deux cahiers qui composent le troisième mémoire consacré au sujet sous le titre École d'architecture rurale ${ }^{8}$. Le texte, d'une centaine de pages, et les dessins (16 planches) s'appuient sur l'expérience de l'auteur -l'architecteentrepreneur François Cointeraux ${ }^{9}-$ d'abord acquise aux côtés de son grand-père maitre-maçon, puis au cours de sa carrière de constructeur. François Cointeraux insiste également sur ses voyages spécialement entrepris «à l'effet de reconnoître dans les villages les divers usages de bâtir, et les matériaux particuliers qu'on y employe » (1790 : 22). C'est ainsi qu'il découvre une "autre méthode de faire le pisé », qu'il présente dans son premier cahier et distingue de la «méthode du Lyonnois » sous les termes de « méthode du Bugey $»^{10}$.

6 À la suite de Cointeraux, c'est encore un architecte d'origine lyonnaise, Jean-Baptiste Rondelet, qui produit un nouveau texte de sept pages sur le sujet, accompagné de deux planches (1802, t. I, $)^{11}$. Fils d'un maçon lyonnais, Rondelet s'appuie aussi sur son expérience pratique personnelle, de même que sur les textes antérieurs qui sont à sa disposition. 


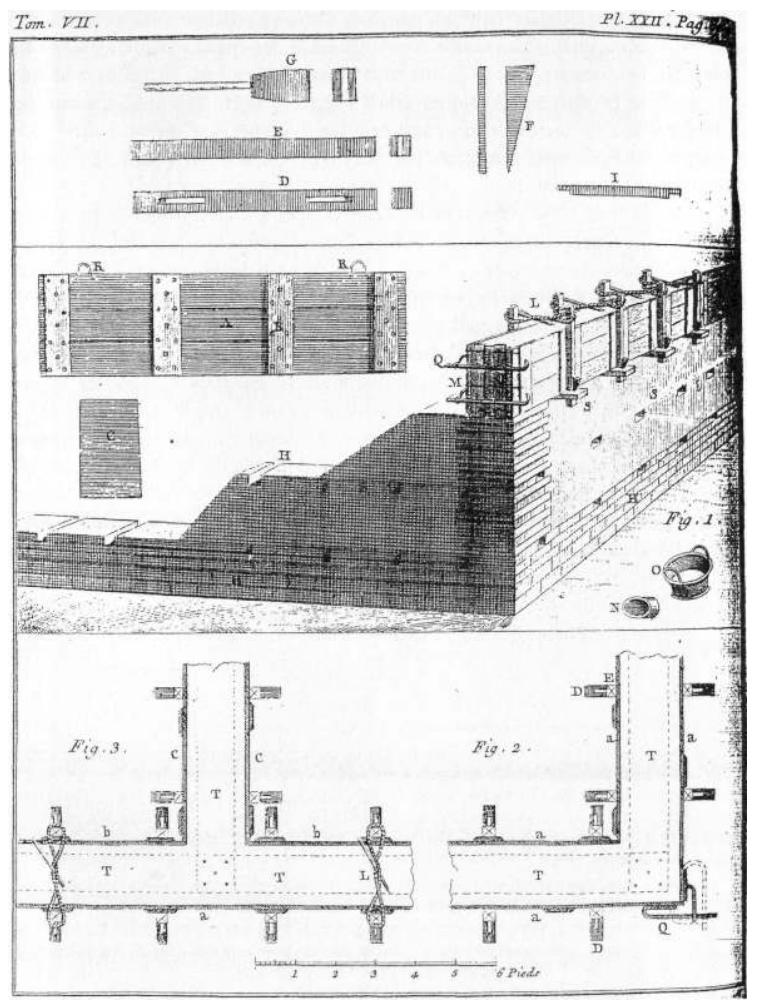

Figure 1. "Pisai ou pisé », « Terre battue entre deux planches, au moyen de laquelle on construit les murs des maisons » (d'après l'Abbé Rozier 1793 : t. VII et p. 659)

7 Ces quatre premières enquêtes ont en commun d'établir une "théorie-pratique ${ }^{12} \mathrm{du}$ pisé. La pratique est élevée par "la voie méthodique » au rang de "noble science ", d'«art de l'incombustibilité». La technique est désormais distinguée des autres manières de bâtir en terre crue, distinction qui n'était pas clairement établie par tous si l'on en juge par le commentaire de François Cointeraux (1790 : 11-12) :

«Qu'on ne s'y trompe pas! le pisé est bien différent de ces misérables constructions faites en terre pêtrie ou en boue, mêlée avec de la paille ou du foin, que bien des personnes confondent avec cet art précieux. J'ai vu même d'habiles gens ne savoir ou ne vouloir pas distinguer cette noble science d'avec la routine que l'on a dans la campagne d'élever quelques murs avec la terre pêtrie ; construction on ne peut plus vicieuse, puisqu'elle ne se soutient qu'autant qu'on lui donne un talus rapide ou une forme bien pyramidale. "

Les règles de «l'art du maçon piseur» se constituent à partir non pas des commentaires existants mais d'une sélection de pratiques observées dans le Lyonnais ${ }^{13}$. Les termes sont choisis et limités à un ou deux synonymes. Ainsi, émergent les mots aujourd'hui communs de "bancher, banché, banchée ", utilisés dans la technique du béton.

\section{De la « théorie-pratique » à la pratique du pisé}

9 Les règles du pisé en main, de nombreux constructeurs de bâtiments ruraux, architectes, ingénieurs, vont tenter de tirer parti des ressources de la technique. Ce qui donne lieu à de nombreuses publications diffusant à leur tour les principes du pisé et rendant compte des expériences qui visent à les mettre en œuvre comme à les améliorer (voir par exemple Champ 1795). Cointeraux produit lui-même une dizaine de 
mémoires sur ce qu'il nomme le « nouveau pisé ». Le capitaine du Génie Frossard étudie en 1844 une manière d'adapter ce mode de construction aux murs des fortifications (vol. 14 : 236-240 et pl. XI, fig. 9). L'ingénieur Oppermann fait expérimenter en 1859 des "maçonneries de terre cimentée " aux fermes impériales de Chalon (Opperman \& Marini 1859 : 135). Le pisé ouvre la voie à la construction en béton; François Coignet, entrepreneur d'origine lyonnaise, élabore son premier béton dit « béton-pisé » à partir d'un mélange de terre et de chaux damée.

Mais on voit bien que la description ne donne pas les clés du savoir-faire. Une chose est de connaître les prescriptions du pisé, une autre est de savoir les mettre à exécution. Le comte Des Garets, sous-préfet de Montteuil-sur-Mer (Pas-de-Calais), lui-même originaire du Beaujolais et auteur d'un essai destiné à substituer le pisé au torchis et à la bauge dans le nord de la France (1823) ${ }^{14}$, fait construire une "maison modèle en pisé » dans le Pas-de-Calais, mais par un ouvrier de l'Ain et selon les plans d'une maison du Beaujolais. C'est ce «maître-ouvrier » qui forme les quatre « ouvriers-piseurs » qui sont ensuite proposés à ceux qui veulent se lancer dans la construction en terre. Sans ouvriers piseurs, les règles de l'art de piser sont difficilement applicables.

11 Même constatation dans le sud-ouest de la France où la bauge, mais aussi un certain type de pisé, étaient alors répandus. Saint-Félix-Mauremont, propriétaire terrien et agronome (1858: 80) jugeait ainsi inapplicable le pisé lyonnais :

" Il est malheureux que, malgré des demandes réitérées, on n'ait pas fait venir à Toulouse, et dans la région du sud-ouest de la France, en Languedoc, en Guienne et en Gascogne, de bons ouvriers piseurs ; car les ouvrages de ce genre que l'on y construit, appelés parois, sont défectueux. Cela provient de plusieurs causes. On néglige de construire en maçonnerie à bain de chaux les fondations et le soubassement; les moules dans lesquels on foule la paroi n'ont point la résistance ni la bonne exécution des banches du pisé; ce qui exige nécessairement une surépaisseur considérable; la terre n'est frappée qu'a la batte, sans employer le pisoir ; aussi ce genre de construction est-il dédaigné. »

12 Comme le montre ce passage, la construction en pisé pratiquée dans le Sud-Ouest était éloignée de la manière lyonnaise. On trouve cette technique désignée localement dans les textes par de nombreux synonymes: pisé, paroi, paroi de tapie, paroi de bâtisse ou muraille. Notons que le mot tapie rappelle le mot d'origine berbère al-tabiya transcrit en espagnol sous le mot tapial ou tabiya.

13 Les mémoires et articles locaux ne permettent pas de bien comprendre ce qu'était le pisé du Sud-Ouest car ils ne s'emploient pas à décrire les parois de tapie locales mais le pisé lyonnais. C'est le cas des deux principaux manuels de construction et d'architecture régionaux: Architecture rurale, Théorique et pratique (1820) et Le Propriétaire architecte (1827). Saint-Félix-Mauremont, qui s'était pourtant fixé comme but de décrire « la qualité des matériaux employés dans les constructions et le prix de la main-d'œuvre dans le Midi de la France» de manière à compléter le Traité d'architecture rurale (1810) de Perthuis, renvoie aux essais de François Cointeraux ${ }^{15}$, tout en donnant les termes employés par l'abbé Rozier et par Rondelet ${ }^{16}$ et en recopiant mot à mot plusieurs passages du Traité de l'art de bâtiri ${ }^{17}$. Deux articles consacrés à la construction des murs de clôture en terre, parus dans des revues locales en 1828 et 1829, donnent peu d'informations sur les savoir-faire liés au damage et au banchage de la terre ${ }^{18}$. Seul un article sur les constructions rurales détaille les moyens de mettre le pisé à l'abri des intempéries et donne une description de la manière de faire les fenêtres (Vicomte de Caumon 1841 : 129-137). 
14 Ainsi les auteurs régionaux jugent-ils la technique en fonction des descriptions données par les Lyonnais et constatent-ils son infériorité. Les défauts l'emportent sur les avantages économiques: "On reproche à ce genre de construction de prendre beaucoup d'espace, d'être accessible aux rats, et de ne pas garder l'enduit de mortier » (Vicomte de Caumon 1841 : 134), l'auteur poursuit :

«Les entrepreneurs de ces sortes d'ouvrages ne cherchent qu'à aller vite; ils ne battent le pisé qu'à moitié ; emploient souvent la terre trop humide ou trop forte, ce qui fait que le mur se fend, se gerce dans les fortes chaleurs et n'acquiert aucune consistance. Des murs en pisé bien faits, ainsi qu'on les construisait du temps de nos pères, deviendraient durs comme de la pierre et dureraient des siècles s'ils étaient abrités » (Vicomte de Caumon 1841 : 132).

Mais le pisé n'apparaît pas seulement comme une technique réalisée avec moins de soin que dans la région lyonnaise ; les contrats de construction montrent qu'il désigne aussi quelquefois une autre pratique (fig. 2). Le terme est ainsi parfois synonyme de paillebard ou bouzillage, c'est-à-dire d'une technique qui se rapproche de la bauge.

Par exemple, un contrat de construction de 1854 décrit ainsi :

« Les murs en pisé ou paillebart auront une hauteur de deux mètres non compris les fondations qui seront de trente centimètres. Leur épaisseur sera de quarante centimètres au couronnement et de soixante centimètres à la base. [...] le chapeau sera en bande de bruyère d'une épaisseur de dix centimètres assujettie par une couche de paillebard de vingt centimètres d'épaisseur ${ }^{19}$.
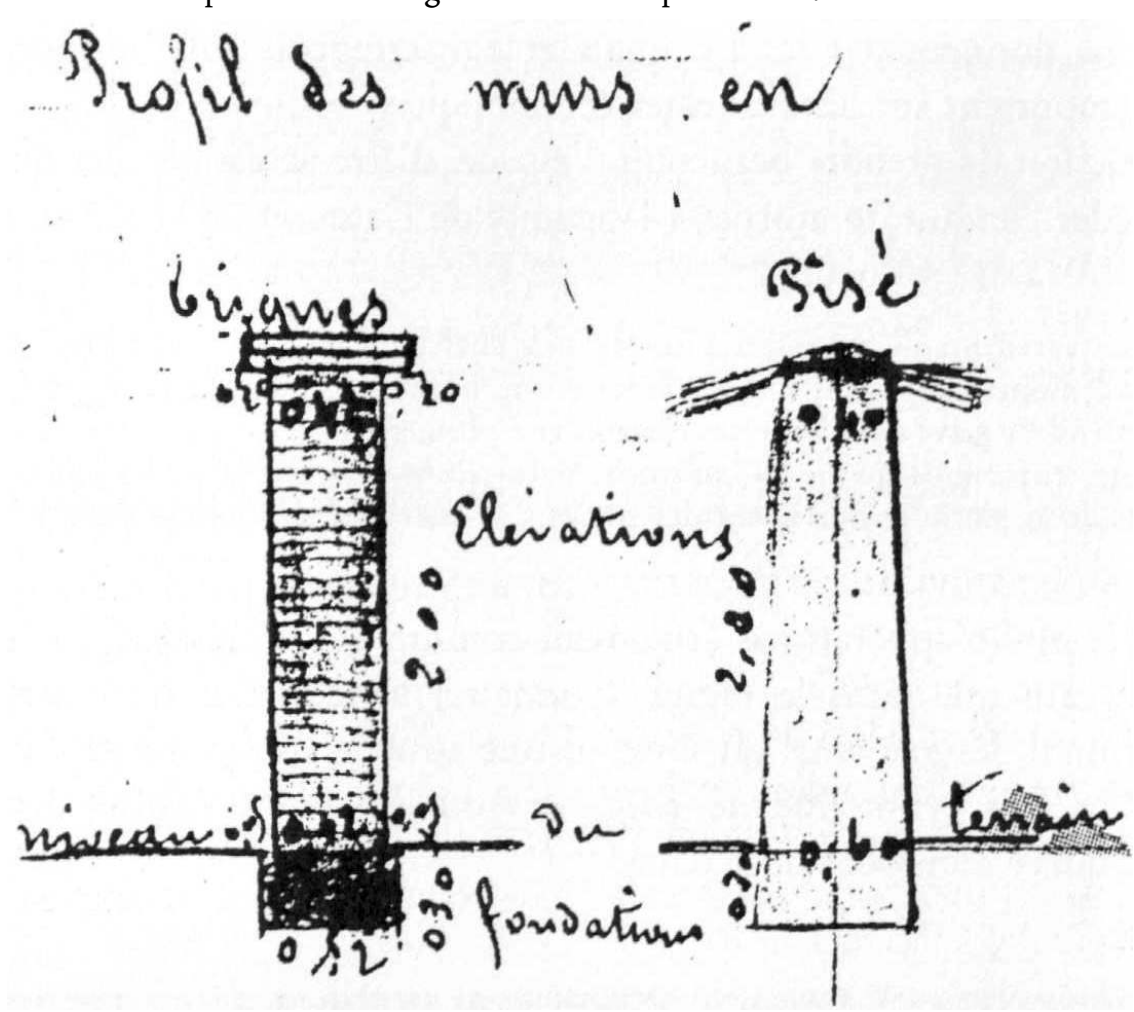

Figure 2. Mur de clôture en terre empilée du jardin de la mairie de Beauzelle (Haute-Garonne) ; d'après un devis de construction de 1854. La technique est désignée sous le terme de " pisé » [ADHG, 20 115]

Le pisé est de même rapproché du paillebart dans les Usages locaux du Tarn :

«On nomme paillebart une espèce de pisé dans lequel ce ne sont pas de petites pierres mêlées à la terre qui maintiennent la cohésion, mais bien de la paille pétrie avec. » (Clausade $1843: 73$ ). 
L'auteur continue :

«Si on les fait en terre non moulée, pisé ou paillebart, ils ont une épaisseur de quarante-quatre centimètres au moins (deux empans environ). Dans ce dernier cas, on les bâtit même en talus de deux côtés, de telle sorte que la partie supérieure a moins d'épaisseur que l'inférieure ".

19 Le mot paroi, qui désigne le pisé, apparaît parfois comme synonyme aussi de torchis. Comme le torchis, la terre battue était en tout cas du ressort des charpentiers. Deux marchés pour la construction de maisons situées dans les environs de Toulouse datant de 1770 et 1777, qui prescrivent respectivement des « Parois de terre batue de 16 pans de haut avec fondation » et des " Paroi de tapié de 16 pans de haut au-dessus du sol ", sont passés avec des charpentiers ${ }^{20}$. Les devis indiquent bien par ailleurs qu'il s'agit de murs construits avec du fruit : « 4 pans à la base, l'épaisseur diminuera en montant» pour le premier et « 4 pans et demy à la base ; 2 pans et demy en haut » pour le second.

Si l'observation des édifices existants montre que le pisé banché existe bien dans la région $^{21}$, les textes permettent donc de comprendre que cette technique était variable et mal différenciée de la technique de construction en terre crue battue mêlée à de la paille, dite paillebard ou bouzillage. Le mot pisé désignait parfois de simples couches de terre battue armée avec de la paille. Les marchés de construction montrent clairement que les murs étaient construits avec un fruit important. Orientés à l'Ouest ou au Nord, ils étaient souvent protégés par des haies de laurier ${ }^{22}$.

21 Alors que le pisé n'est pas précisément décrit et défini par les auteurs locaux, en revanche, le paillebard, souvent confondu avec le pisé, l'est. C'est au chapitre " Mortier » de ses Us et coutumes de la ville de Toulouse que l'architecte et ingénieur de la ville, Pierre Lebrun (1753 : 45-46) donne sa composition : « La Bauge ou Paillevard se compose avec du sable \& de la terre noire, autant de l'un que de l'autre, dans laquelle composition on mêle de la paille ou du gros foin ». Saint-Félix-Mauremont le définit comme «un mélange de terre argileuse avec de la balle de blé ou de la paille hachée, que l'on place tout frais avec une houe, une pioche ou un trident, en l'affermissant, en en régularisant ses parements, et dont les premières couches sont appuyées dans une rigole qui lui sert de fondation » (Saint-Félix-Mauremont $1858: 80$ ).

La technique consistait à empiler la terre mêlée de paille par couches puis à régulariser les parois à l'aide d'un outil tranchant. Dans le cas de mur de clôture, la partie supérieure des murs était recouverte d'un chaperon réalisé avec des branchages disposés en débord par rapport au mur. La terre était prise sur place, au pied des murs :

«Le paillebart n'est réellement qu'un véritable torchis; il exige une plus forte épaisseur que le pisé, et une bien plus grande quantité d'eau. On le compose avec de la terre grasse détrempée et mêlée avec de la paille ; cette opération se fait au pied même de la muraille qu'on élève. On l'établit par couches horizontales d'environ 8 pouces d'épaisseur [21,6 cm]. L'ouvrier se sert pour cela d'un hoyau à deux fourchons, au moyen duquel il enlève la quantité de terre nécessaire, et la dépose sur le mur en lui faisant éprouver un tassement au moyen d'un coup de hoyau " (Vitry 1829 : 394).

Les contrats de construction montrent que les murs avaient toujours du fruit : pour un mur de deux mètres, environ $75 \mathrm{~cm}$ d'épaisseur dans les fondations et $45 \mathrm{~cm}$ dans la partie haute. Une description en est donnée dans un devis de 1844 :

«Les dits bouzillages seront composés avec de la paille, chaume ou autres matières de la même nature, très bien mélangés avec de la terre et de l'eau, et seront établis par couches horizontales de vingt à vingt cinq centimètres de hauteur 
conformément aux dimensions prescrites précédemment; néanmoins il est expressément réservé qu'on sera obligé de donner un excédent de dix centimètres de plus de largeur afin qu'après que les dits bouzillages seront terminés on aura soin de couper avec une bêche ou autre outil tranchant ces excédents de largeur, et cela pour éviter qu'il n'existe pas de flaches ni aucune difformité dans la construction. [...] dans le délais de deux mois au plus tard, l'entrepreneur sera obligé de les couvrir avec de la bruyère de bonne qualité [...] à raison de trois fagots par mètre courant ou de longueur lesquels seront assujettis au moyen de piquets en bois de chêne de soixante centimètres de longueur et de cinq à six centimètres de diamètre. Ensuite la dite bruyère sera couverte avec de la terre grasse préparée à cet effet, qui formera le couronnement et sera placée avec soin $»^{23}$.

Il est paradoxal de voir un type d'ouvrage aussi rudimentaire exposé avec autant de détails. Cela indique sans doute qu'aussi simples qu'ils puissent être, les bouzillages étaient des ouvrages très variables, laissés à l'initiative de ceux qui les construisaient. La préparation de la terre, l'épaisseur des couches, le fruit du mur différaient d'un endroit à l'autre. L'architecte Urbain Vitry rapportait ainsi (1829: 395) qu'il avait vu mêler de la terre avec du chiendent pour économiser la paille. Les végétaux qui servaient de chaperon étaient de même extrêmement variés : « poignées ou manettes de bruyères, brucs, joncs, broussailles, sarments » (Saint-Félix-Mauremont 1858 : 80). Ceux qui réalisaient ces ouvrages n'étaient pas forcément des ouvriers du bâtiment. Dans un marché de 1787, la reconstruction du mur de clôture en paillebart d'une métairie située à Saint-Cyprien (Toulouse) était assurée par un "travailleur de la paroisse », un manœuvre qui n'était donc pas un ouvrier spécialisé24.

Saint-Félix-Mauremont (1858: 80) jugeait cette technique « encore plus rustique que le pisé ", et donnait comme deuxième signification au mot bousillage, dans son Vocabulaire universel des termes d'architecture, celle de " mauvais ouvrage $»^{25}$. Il reprenait en cela une définition courante du mot. Le Dictionnaire d'architecture de Quatremère de Quincy (1788 : 315) attribue en effet deux significations au mot : « maçonner avec du chaume \& de la terre détrempée » et "faire quelque ouvrage sans soin, sans exactitude, sans propreté ». Aujourd'hui encore, le terme désigne vulgairement l'action d'abîmer ou de casser.

Le Vicomte de Caumon, constructeur de bâtiments ruraux à ses heures, lui reconnaissait cependant une « solidité étonnante » :

«Le peuple se sert fréquemment de ces sortes de murs pour la construction de ses modestes habitations et tout le monde les emploient pour clore les jardins, vignes et autres lieux, où ils sont de longue durée si on les tient bien couverts, et surtout si la terre dont on se sert est d'une nature boulbéneuse*. Lorsqu'ils sont bien faits, ils ont une solidité étonnante, même à la hauteur de cinq à six mètres " (1841:135).

C'est sous forme de mur de clôture que la technique était la plus répandue. À Toulouse, la maçonnerie de terre crue était autorisée pour clore les jardins. Ce type de murs est aussi décrit dans les Usages Locaux (1843) du département du Tarn :

«Dans tous ces lieux, une hauteur uniforme et non contestée est presque toujours admise pour les clôtures qu'on construit à l'amiable : c'est celle de deux mètres, chaperon compris ; c'est à peu près ce qu'on nommait la toise ou neuf empans » (Clausade $1843: 65)$.

Ce que révèlent surtout les contrats de construction, c'est que l'avantage principal du paillebart était son prix: trente fois moins cher que la maçonnerie de brique. La comparaison est spectaculaire: le bouzillage valait, en $1844,0,78 \mathrm{fr}$. le mètre cube 
( 0,80 fr. en 1854$)$; la maçonnerie de briques foraines entières $26,40 \mathrm{fr}$. Le pisé était évalué en 1841 à 0,80 ou 0,90 fr. le mètre cube.

\section{Le rôle des «figures »}

Dans la «voie méthodique »-la démarche qui permet de "réduire» la technique en «art du pisé »-, les illustrations jouent un rôle fondamental. Les quatre premiers essais sur le pisé s'accompagnent tous de dessins réalisés spécifiquement pour illustrer le texte auxquels ils se réfèrent. L'illustration, qui se compose au départ, selon la tradition de la Description des Arts et Métiers (Jaoul \& Pinault 1982), d'un dessin à la fois facilement compréhensible (une vue perspective) et de dessins plus abstraits (géométraux) conservant les proportions et les dimensions (notamment des outils), évolue vers une représentation plus abstraite combinant les projections horizontales et verticales. Ainsi l'architecte Boulard reprend, en 1786, la planche de Goiffon (1772) en la complétant par deux projections horizontales (plans) du dispositif de banchage. Parce que «les choses les plus simples sont difficiles à comprendre, lorsqu'on ne les a jamais vues", Cointeraux (1790:9) multiplie les planches (pas moins de seize pour ces deux premiers « cahiers »). Le matériel et les outils sont représentés par des projections mises en correspondance: vues dites "à plat ", «sur le dos ", "par-dessus », "par côté ", "en face ", "vue couchée». De la sorte, les quatre faces du moule sont dessinées, le pisoir est représenté en trois dessins et non un seul, comme dans les mémoires précédents : en perspective, en vue de côté et de dessus.

De tels dessins semblent avant tout destinés à des architectes ou à des lettrés. Dans le cas de Cointeraux, fils d'entrepreneur, on peut imaginer que le travail de « réduction en art » de la technique du pisé soutient autant son aspiration à dialoguer d'égal à égal avec les véritables théoriciens de l'art de bâtir que son désir de favoriser l'innovation. Mais parce qu'il est un homme à la rencontre de la pratique et de la théorie, comme il aimait à le revendiquer, Cointeraux a conscience des limites des dessins, "vérités palpables et constantes ${ }^{26}$, puisqu'il tente d'en augmenter la lisibilité en les colorant et va même jusqu'à les compléter par la mise en vente de maquettes en bois destinées à construire «les outils du pisé $»^{27}$. La connaissance par le dessin est complétée par la connaissance par le «modèle », par un enseignement qui n'est plus dans l'analytique mais dans l'analogie.

31 Le rôle primordial joué par l'iconographie se mesure aussi au nombre de planches et de dessins recopiés tantôt à l'identique, tantôt avec variantes, dans les nombreux mémoires qui divulguent le pisé lyonnais au XIX ${ }^{\mathrm{e}}$ siècle. Le comte de Lasteyrie reprend dans sa Collection de machines, d'instrumens, ustensiles, constructions $(1820)^{28}$, la planche X de l'École d'architecture rurale de Cointeraux (1790 : fig. 2). Le dessin est retravaillé, mais est clairement inspiré de la planche présentant la «méthode du Bugey » consistant à planter de grands poteaux évitant de démonter à chaque assise le système de banchage. Les deux traités de construction du Sud-Ouest précédemment cités reproduisent la planche du Cours complet d'agriculture (1786) de manière presque identique (fig. 3 et 4). Le rendu n'est plus ombré mais au trait. Seul le pisoir représenté en perspective est inspiré du Traité de l'art de bâtir de Rondelet (1807-17, vol. pl. IV, fig. 8). Les dessins montrent ainsi d'emblée ce que l'on comprend à la lecture des textes : les deux pages consacrées au pisé ne se basent pas sur une enquête locale ou des sources de première main, mais sur une publication existante. 


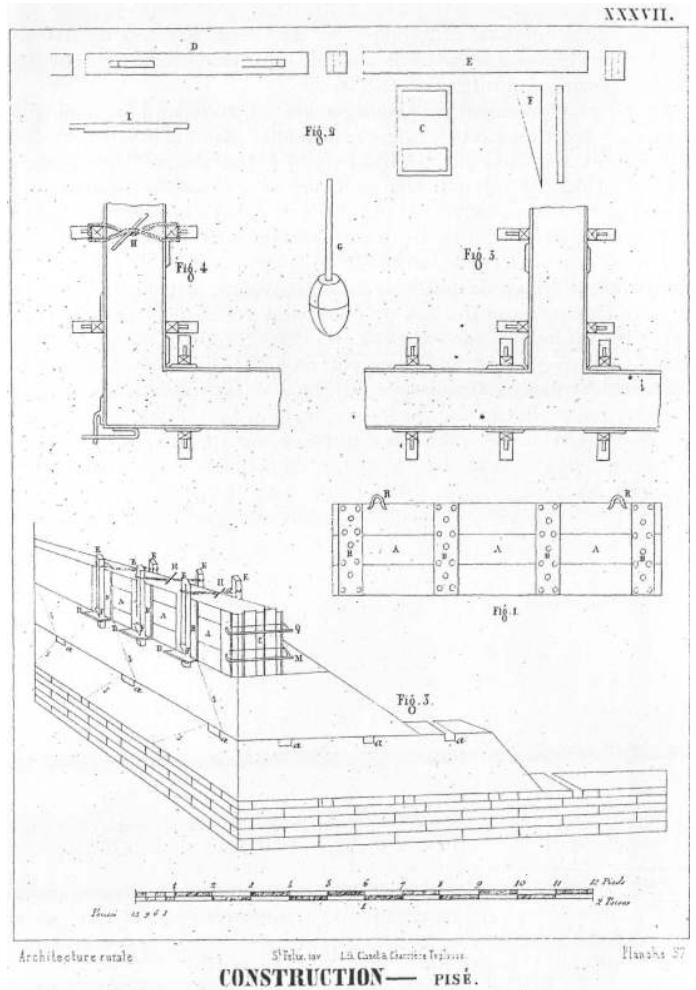

Figure 3. "Construction - Pisé » (Saint-Félix-Mauremont 1793) La planche du Toulousain reprend la planche XXII du tome VII du Cours complet d'Agriculture (L'Abbé Rozier 1793)

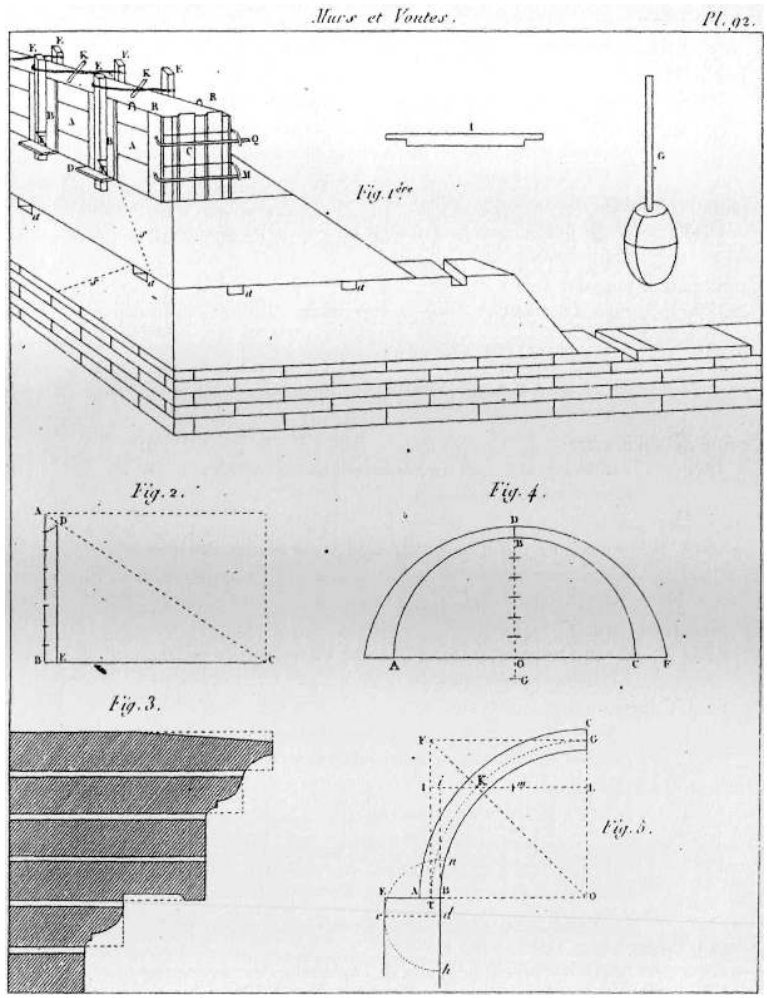

Figure 4. « Murs et voûtes » (d'après Urbain Vitry 1827 : pl. 92) La planche reproduit exactement le dessin de la planche 6 de l'Architecture rurale (Saint-Felix-Mauremont 1820) 


\section{BIBLIOGRAPHIE}

Champ, J.

1795, Avis aux propriétaires des terres de Hongrie, de la Bohême et de toute l'Allemagne, concernant l'adoption de la manière de bâtir en terre seule massivée, décrite dans le cours d'architecturale rurale, pratique, nouvellement publié, etc. Vienne et Leipsick.

Clausade, Amédée

1843, Usages locaux ayant force de loi et topographie du département du Tarn. Paris : Jolibert.

Cointeraux, François

1790, École d'architecture rurale, premier cahier... Paris : Chez l'auteur.

Comte de Lasteyrie

1820, Collection de machines, d'instrumens, ustensiles, constructions, appareils, etc. employés dans

l'Economie rurale, domestique et industrielle. Paris. (2 vol.).

Comte de Villeneuve

1828, « Murs de clôture en terre », Journal des propriétaires ruraux pour les départemens du Midi :

114-115.

Comte Des Garets

1823, Notice sur le pisé et sur les avantages de son introduction dans les départemens du Nord de la France. Boulogne : imprimerie de Le Roy-Berger (20 p. et 1 pl).

Dethier, Jean (ed.)

1981, Des Architectures de terre ou l'Avenir d'une tradition millénaire. Paris : Centre Georges

Pompidou.

Frossard, Capitaine du Génie

1844, « Note sut la manière de confectionner le pisé en quelques points de l'Algérie », Mémorial de l'officier du Génie (vol. 14).

Goiffon, Georges-Claude

1772, L'Art du maçon piseur. Paris : Le jai.

Jaoul \& Pinault

1982, « la collection “Description des Arts et métiers”. Étude des sources inédites de la Houghton Library, Université Harvard », Ethnologie française 2, 4 : 335-360

Lebrun, Pierre

1753, Les Us et coutumes de la ville de Toulouse, avec les instructions pour connoître les matériaux, et généralement tout ce qui concerne la Bâtisse. Toulouse : B. Pijon.

Liger, Louis

1755, L'Économie générale de la campagne. Paris : Saugrain fils.

Oppermann, Charles-Alfred \& Marini

1859, «Les maçonneries en carreaux de terre cimentée », Nouvelles annales de la construction, août, col. 135-135

Quatremère de Quincy, Antoine Chrysostôme

1788, Encyclopédie méthodique. Architecture. Paris : Panckoucke « Bousiller » et « Bousilleur ».

Rondelet, Jean-Baptiste

1802-1817, Traité théorique et pratique de l'art de bâtir. Paris : Firmin Didot (7 vol.). 
Rozier, Georges-Claude (dit l'abbé Rozier)

1781-1800, Cours complet d'agriculture Théorique, Pratique, Economique et de Médecine Rurale et Vétérinaire, suivi d'une Méthode pour étudier l'Agriculture par Principes, ou Dictionnaire universel d'agriculture. Paris : rue et hôtel Serpente (10 vol.).

Saint-Félix-Mauremont, Armand-Joseph-Marie (de)

1845, Traité historique et descriptif, critique et raisonné des ordres d'architecture. Toulouse :

Douladoure.

1858, Architecture rurale Théorique et pratique à l'usage des propriétaires et des ouvriers de la campagne. Toulouse : Douladoure (1 1 ère édition 1820).

Teyssot, Georges

1981, «L'Architecture en pisé », Revue des Monuments historiques 116 : 32-35.

Vicomte de Caumon

1841, « Des constructions rurales », Journal d'agriculture de la Haute-Garonne, pp. 129-137.

Vitry, Urbain

1829, « Des constructions en terre. Pour les murs d'enceinte et les bâtimens d'exploitation en général », Le Propagateur des procédés industriels pour le Midi de la France : 391-395.

\section{NOTES}

2. L'ouvrage se compose de 57 pages et 1 planche. D'après François Cointeraux, il était épuisé en 1790.

3. Goiffon distingue le terme pisé de ceux qui sont proches mais qui appartiennent à d'autres domaines: «Nos villageois disent comme le portent nos Dictionnaires, piler du sel, piler du ciment, piler dans un mortier avec un pilon, \&c, mais ils disent de plus que nos Dictionnaires, piser la terre autour d'un pieu pour la rendre plus inébranlable. Dans leur entendement, piser, donne l'idée du rapprochement des parties séparées, du rétablissement de leur union avec la masse, de la dureté que cette masse acquiert en devenant de plus en plus compacte, à mesure qu'elle est plus-longtems \& plus fortement frappée par le pison: tandis que piler entraîne celle de la réduction d'une masse dure \& liée en elle-même, en une multitude de parcelles séparées : pétrir, celle de plus d'intimité dans le mélange de plusieurs matières, tant quelles sont dans un état de molesse : fouler celle d'une pression souvent réitérée, \& opérée principalement avec les pieds, àpeu-près comme piétiner : mais aucune de ces idées ne convient à l'action qui donne l'existence à nos murs de terre [...]. » Goiffon (1772: 5, note 1$)$.

4. Catherine-François Boulard (?-1794), architecte de la ville de Lyon, était l'auteur de plusieurs mémoires techniques: Sur les moyens de garantir les canaux et leurs écluses de tout atterrissement (1778); Sur la forme et la nature des jantes pour les roues de voitures (1781), et Quelle serait la voiture de transport la plus forte, la plus légère, la plus roulante et la moins capable de dégrader les chemins, mémoire couronne par l'Académie de La Rochelle.

5. La notice «Pisai ou pisé » est comprise dans le tome VII édité en 1786, pp. 719-737 et pl. XXII, p. 736.

6. «Si M. Goiffon eût pratiqué le pisé, son traité aurait été complet, et seroit la cause que cet art se serait répandu [... ]» (Cointeraux $1790: 4)$.

7. Les sous-titres sont : «Des qualités de la terre à piser; De la préparation de la terre à piser; Du temps propre à former le pisé ; Description du moule \& des outils propres à faire le pisé ; Outil, Construction du pisé ; Couverture des murs en pisé; De l'enduit du pisé \& du crépi appelé rustiquage ; Prix du pisé ; De la conduite du pisé pour la construction d'une maison ; De l'enduit, 
Prix du pisé pour le bâtiment; Conclusion; Démolition du pisé ; Engrais provenant du pisé, Moyen de rendre toute terre propre à faire du pisé ». «Pisai ou pisé » (Rozier 1786 t. VII).

8. Le premier cahier s'intitule : Ecole d'architecture rurale, premier cahier, Dans lequel on apprendra soi-même à bâtir solidement les Maisons de plusieurs étages avec la terre seule, Paris, Chez l'auteur, 1790. In- $8^{\circ}$. Il est composé de $32 \mathrm{p}$. et $12 \mathrm{pl}$. Le second cahier est publié en 1791 sous le titre : Ecole d'architecture rurale, second cahier, Dans lequel on traite : $1^{\circ}$ de l'art du Pisé ou de la Massivation, $2^{\circ}$. Des qualités des terres propres au pisé, $3^{\circ}$. Des détails de la main d'œuvre, $4^{\circ}$. Du prix de la toise, $5^{\circ}$ Des enduits, $6^{\circ}$. Des peintures, Paris, chez l'Auteur, 1791. $\ln -8^{\circ}$. Il comprend 76 p. et $4 \mathrm{pl}$.

9. Sur Cointeraux, voir Teyssot (1981:32-35) et Dethier (1981).

10. Voir « Autre méthode de faire le pisé ou les maisons avec la terre » (1790:22-27 et pl. IX et X). 11. Voir t. I, Chap. II « Des pierres artificielles », art. II « Du pisé », pp. 103-109 et pl. IV et V (éd. De 1871).

12. Cointeraux observe cette « habilité, que l'on pourrait presque nommer théorie-pratique, qui fait que les uns gagnent plus que les autres, lorsqu'ils possèdent, outre la pratique, l'idée scientifique de la construction » $\left(1790,2^{\text {ème }}\right.$ cahier : 45$)$.

13. Les commentaires de Pline et de Varon sont cités, mais non ceux des auteurs «modernes » traitant « d'économie rurale » comme Louis Liger (1755, livre I, chap. III : 56).

14. Voir aussi « Rapport fait par M. Vallot, sur un mémoire de M. Des Garets, relatif à l'emploi du pisé et aux avantages de son introduction dans le nord de la France », Bulletin de la Société pour l'encouragement de l'industrie nationale, 1824, t. 23, pp. 25-29.

15. «Nous renverrons, pour une connaissance plus approfondie, aux ouvrages de M. Cointereaux, professeur d'architecture rurale, qui a perfectionné le pisé ou construction en terre, et inventé les pierres factices ». (Saint-Félix-Mauremont 1820 : 94). Voir le chapitre VII, article $1^{\mathrm{er}}$ «Du Pisé », (pp. 95-99).

16. Le mot lançonnier est issu des textes de Goiffon et Rozier, les mots lanssoniers ou clefs de Rondelet: «Ces banches se placent sur des traverses D, appelées lançonniers, lassonniers ou clefs [...]». (Saint-Félix-Mauremont 1820 : 96).

17. Pour la description du pisoir notamment : «On fait usage du pisoir en le tournant à chaque coup, qui tombe d'un pied de haut, de manière à croiser les traces qu'il imprime sur la couche de terre, et à la massiver également dans toute son étendue ». (Saint-Félix-Mauremont 1820 : 98). Voir le même passage dans le Traité de l'art de bâtir (Rondelet 1802-17 : 107, vol. 1, éd. De 1871).

18. Comte de Villeneuve (1828: 114-115) ; Urbain Vitry (1829 : 391-395).

19. « Devis des travaux à exécuter pour la construction des murs de clôture du préau du jardin de la mairie de Beauzelle ». Le 9 août 1854 [ADHG, 20 115]. Même description au XVIII siècle : «[...] laquelle batisse sera faite de paroits de terre batue, lesquelles paroits auront quatre pans d'epaisseur sur le bas compris le fondement. La ditte epaisseur diminuera en montant suivant l'art, et auront seize pans hauteur compris le fondement [...]». « Contrat de bail de construction de maison au lieu de Lardenne moyennant la somme de $150 \mathrm{fr}$. entre Guilhaume Bacou et André Balancy charpentier habitant Toulouse », 20 avril 1770. Vidal notaire [ADHG, 3E 11990].

20. "Contrat de bail de construction de maison au lieu de Lardenne moyennant la somme de 150 fr. entre Guilhaume Bacou et André Balancy charpentier habitant Toulouse ", 20 avril 1770. Vidal notaire [ADHG, 3E 11990]. [Construction d'une maison et d'un chay sur une pièce de vigne. Philippe Rebelin, maître tailleur d'habits, Pierre Charron, François Martel et Jean Salat, charpentiers à Toulouse]. Jean Monereau-Roc notaire [ADHG, 3E 10790].

21. On observe in situ les dimensions suivantes : soit des couches fines (de 5 à $12 \mathrm{~cm}$ de hauteur), soit des couches épaisses (de 15 à $40 \mathrm{~cm}$ ). Alain Klein, Etude sur l'architecture rurale en terre crue de Midi-Pyrénées, rapport dactyl., DRAC Midi-Pyrénées, 1993.

22. «Dans les environs de Toulouse, des murs épais, formés de terre battue, garantis à l'ouest et au nord par une haie de laurier, résistent souvent pendant des siècles à toutes les intempéries, à 
toutes les causes de destructions ». "Variétés », Gazette du Languedoc, jeudi 30 janvier 1845, $\mathrm{n}^{\circ} 2702$.

23. "Devis des travaux et fournitures à faire pour la reconstruction des bouzillages qui doivent être établis pour clore le cimetière de la ville de Grenade ». Antoine Claverie, géomètrearpenteur patenté, Grenade, le 28 août 1844 [ADHG, 20 582].

24. [Bail à besogne). Reconstruction en paillebart des parois de clôtures qui bordent la métairie de Bordebasse, hors de la porte Saint-Cyprien, 20 mai 1787. Jean Salut notaire [ADHG, 3E 11990].

25. Ce Vocabulaire est compris dans son Traité historique et descriptif $(1845: 14)$.

26. Il écrit dans un de ses ouvrages : «Ce petit ouvrage n'offre pas seulement des raisonnements, des préceptes, et les plus minutieux détails, mais encore des figures gravées avec leurs descriptions, comme présentant aux yeux et à l'esprit des vérités palpables et constantes". François Cointeraux, Supplément du cours complet d'agriculture de l'abbé Rozier, Paris, Cointeraux, 1809. In- $8^{\circ}, 56 \mathrm{pl} .8 \mathrm{p}$.

27. François Cointeraux vendait ainsi trois maquettes avec leurs descriptions imprimées. Un « modèle des outils de l'ancien Pisé » ( 5 fr.), des « modèles des moules du nouveau Pisé » (5 fr.), et un « modèle des Voûtes en Pisé » (5 fr.).

28. Voir vol. 1, pl. VII, fig. 1. «Manière de puiser l'eau dans un puits sans descendre d'un étage élevé ; fig. 2. Construction en pisé ; fig. 3. Vase oblong pour porter le mortier ; fig. 4. Potence à échafaudage ». "Les appareils qu'on emploie généralement pour construire en pisé diffèrent de ceux que nous présentons ici. Nous avons cru qu'il serait d'autant plus intéressant de décrire ces derniers, qu'ils ne sont pas connus, et qu'ils sont plus simples et moins dispendieux que les autres. Nous les avons vus employés dans le département de l'Isère », ibid., p. 2.

\section{RÉSUMÉS}

La construction en terre crue damée entre des planches, dite pisé, est une technique de construction rudimentaire pratiquée en France, notamment autour de Lyon et de Toulouse. Elle a fait l'objet, dans la seconde moitié du XVIII siècle, d'une série d'enquêtes qui débouchent sur plusieurs publications savantes. Un corpus de textes et d'images tendant à rassembler les savoirs épars et non écrits, à les mettre en ordre méthodiquement, se constitue de manière à établir et promouvoir «l'art du maçon piseur». Parmi le nombre relativement important d'articles, de commentaires et de mémoires publiés aux XVIII et XIX ${ }^{e}$ siècles, émergent quatre essais rédigés par des Lyonnais qui codifient les règles de "l'art du pisé". Ces essais suscitent à leur tour une série de publications qui s'emploient à divulguer et appliquer les normes du pisé lyonnais.

A rudimentary construction technique with tamped mud between planks, called "pise", was used in France in the Lyon and Toulouse areas. Several investigations on the subject led to scholarly publications. A corpus of texts and images tried to bring together sparse and oral knowledge and to put it in order, to promote the "art of the bricklayer". Among the numerous articles, commentaries and thesis published during the $18^{\text {th }}$ and $19^{\text {th }}$ centuries, four essays (written by authors from Lyon) came out which codified the rules of the "art of pisé". These essays led to several other publications in order to spread and apply norms laid down by the Lyonese.

La construcción con tierra cruda y apisonada entre tablas, llamada pisé, es una técnica rudimentaria. Fue practicada en Francia, especialmente en las comarcas de Lyon y de Toulouse. 
Durante la segunda mitad del siglo XVIII, di lugar a una serie de encuestas que sirvieron de base para varias publicaciones eruditas. Un conjunto de textos y de imágenes que tendían a recoger los saberes esparcidos y no escritos, fue constituido con el fin de establecer y de promover «el arte del albañil piseur (i.e. que construye con pisé)». Entre los artículos, comentarios y memoriales, en cantidad relativemente importante, publicados en los siglos XVIII y XIX, sobresalen cuatro ensayos, redactados por Lyoneses, que codifican « el arte del pisé ». A su vez, estos ensayos suscitaron textos que se dedican a divulgar y amicar las normas del pisé de Lyon.

INDEX

Mots-clés : architecture, construction rurale, pisé, France, terre crue

\section{AUTEUR}

\section{VALÉRIE NÈGRE}

CDHT, CNAM, 5 rue du vert bois, 75003 Paris/École d'Architecture de Strasbourg 